\title{
Effect of passive whole-body rotation on sound localization accuracy of listener subjective straight ahead
}

\author{
Akio Honda $^{1, *}$, Yoji Masumi ${ }^{2, \dagger}$, Yôiti Suzuki ${ }^{2}$ and Shuichi Sakamoto ${ }^{2}$ \\ ${ }^{1}$ Department of Information Design, Faculty of Informatics, \\ Shizuoka Institute of Science and Technology, \\ 2200-2, Toyosawa, Fukuroi, 437-8555 Japan \\ ${ }^{2}$ Research Institute of Electrical Communication, Tohoku University, \\ 2-1-1 Katahira, Aoba-ku, Sendai, 980-8577 Japan
}

\begin{abstract}
This study investigated the effect of passive whole-body rotation on the accuracy of listener subjective straight ahead. Listeners sat on a digitally controlled spinning chair placed at the center of a circular loudspeaker array (radius $=1.1 \mathrm{~m}$, speaker spacing $=2.5^{\circ}$ ) and were exposed to a single 30-ms pink noise burst emitted from one loudspeaker of this array. Under the chair-still condition, listeners were asked to keep their head still, whereas under the chair-rotation condition, listeners were asked to keep their head still and their chairs were rotated at angular velocities of 5, 10, and $20 \%$ s. In both cases, listeners judged whether the stimulus was presented from the right or left of the subjective straight ahead, and there was a significant decrease in the sound localization accuracies under the chair-rotation condition, while chair rotation speed had almost no effect on sound localization accuracy.
\end{abstract}

Keywords: Listener movement, Sound source localization, Rotation speed, Spatial hearing, Multisensory integration

PACS number: 43.66.Qp [doi:10.1250/ast.41.249]

\section{INTRODUCTION}

Spatial hearing is a multisensory integration process that also includes self-motion [1]. In fact, head movement is known to facilitate sound localization and dynamically changes the information input into ears [2-8]. However, recent studies have shown that sound localization accuracy deteriorates during head rotation [9-11], which has inspired several controversial studies on the effects of listener movement on sound localization.

To resolve the inconsistency of the obtained results, conditions resulting in the deterioration of sound localization accuracy should be further accumulated by detailed investigation of sound localization aspects during listener motion. In contrast to previous works [9-11], which have dealt with listener active rotation, we decided to study the effects of listener passive movement on sound localization accuracy. In doing so, we herein investigated the auditory subjective straight ahead (SSA) of listeners, which means the perceived orientation to be directly in front of the

*e-mail: akio.honda6@gmail.com

${ }^{\dagger}$ Current affiliation: Rion Co., Ltd. listener, when they passively rotated at relatively low angular velocities of 5-20\% s. In particular, SSA judgment was chosen for the sake of experiment simplicity, since this judgment is based on the comparison between a sound image percept and the internal basis of straight ahead, which allows one to use a one-interval scheme. Conversely, the minimum audible angle (MAA) judgment is based on the comparison between the percepts of two sound images and therefore requires the use of a two-interval scheme. Moreover, humans' azimuthal sound localization is most accurate for straight ahead, as MAA shows the smallest values in this direction [12].

The passive body movement of listeners is known to influence their auditory space perception. For instance, when blindfolded listeners are passively rotated around a vertical axis, they perceive a physically stationary sound as displaced in a direction opposite to their self-rotation, which is known as auditogyral illusion [13]. Recently, Lewald and Karnath [14] investigated the effect of passive whole-body rotation around the earth-vertical axis on the lateralization of dichotic sound, showing that the auditory median plane of the head was shifted in a direction opposite to that of rotation, which indicated that the 
intracranial auditory perception was shifted in the rotation direction. In addition, Teramoto et al. [15] demonstrated that passive vestibular linear acceleration distorts auditory space perception. However, the effects of passive wholebody rotation on the sound localization accuracy of listener auditory SSA have not been extensively investigated.

The perception of body orientation in space depends on the multisensory evaluation of visual, vestibular, and proprioceptive sensory inputs [16]. In fact, a deviation of the SSA in pointing tasks is observed when vestibular stimulation can induce a distortion of egocentric coordinates [16]. Moreover, in cases when normal listeners show quite accurate judgments of the auditory SSA, the judgments of patients with hemineglect or hemianopia exhibit large deviations [17]. Nevertheless, the effects of passive rotation on auditory SSA remain unclear, and this work can therefore contribute to a better understanding of spatial hearing mechanisms and the multisensory integration process.

We also investigated the effects of rotation velocity on SSA accuracy. Previous studies showed that the sound localization accuracy is reduced during active head movement [9-11], particularly if this movement is rapid. However, Honda et al. [11] demonstrated that this phenomenon is only weakly dependent on movement velocity. These findings suggest that the deterioration of sound localization accuracy is commonly observed not only for rapid but also for moderate or slow head movement. However, only few studies investigated the effects of rotation velocity for listener passive movement. Therefore, we herein probed the effects of passive wholebody rotation on sound localization accuracy in terms of SSA judgment.

\section{METHOD}

Eight (four male and four female) undergraduate and graduate students with normal hearing (hearing level $<20 \mathrm{~dB}$ ) aged 20 to 25 (mean $(M)=20.8$ ) participated in the experiment. Prior to the experiment, we checked whether listeners showed finite slope values of psychometric functions using a short sound localization session.

Figure 1 illustrates the employed experimental setup. All listeners were tested individually in a dark anechoic room. Sound was emitted from a 30-mm loudspeaker (Hosiden $02547 \mathrm{~N} 101$ ) installed in a plastic cylinder of $53 \mathrm{~cm}^{3}$ filled with an absorbing material (polyester nonwoven fabric). A light-emitting diode (LED) was attached to the top of the loudspeaker located at the center of the loudspeaker array $\left(0^{\circ}\right)$. The sound stimulus was presented from a loudspeaker of a circular array (radius $=1.1 \mathrm{~m}$ ) consisting of 37 loudspeakers placed at an angular spacing of $2.5^{\circ}$. The sound stimulus was a $30-\mathrm{ms}$ pink noise burst including 5-ms raised cosine rise and decay parts. The

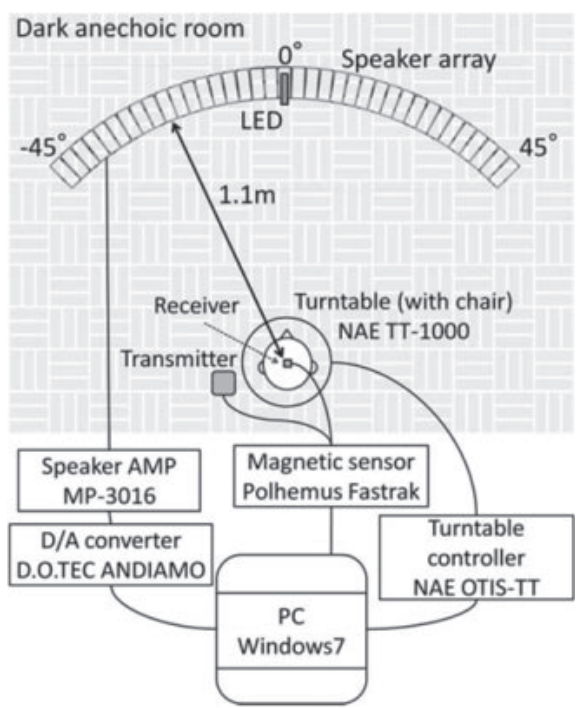

Fig. 1 Outline of the loudspeaker array system.

A-weighted sound pressure level was set to $70 \mathrm{~dB}$ when the steady part of the sound stimulus was presented as a continuous, steady pink noise. In this experiment, listeners were asked to report whether the sound stimulus was presented from the left or right of the SSA (two-alternative forced choice, 2AFC). The experiment was conducted under chair-still and chair-rotation conditions, which were tested separately and in a counterbalanced way between listeners.

Under the chair-still condition, listeners were asked to sit on the chair facing $0^{\circ}$ and gaze at the LED, which was lit for one second. Listeners were asked to keep their head still while the LED was lit. After the LED was turned off, a sound stimulus was presented from one loudspeaker randomly selected from the seven loudspeakers located within $\pm 7.5^{\circ}$ of the listener physical straight ahead. Listeners were asked to judge whether the sound stimulus was presented from the left or right of the SSA. The chairstill condition comprised one session and 140 trials in total (seven loudspeaker positions $\times 20$ trials). Loudspeaker positions were presented in fully randomized order. Before test trials, listeners completed 35 practice trials.

Under the chair-rotation condition, listeners were asked to sit on the chair facing $0^{\circ}$ and gaze at the LED, which was lit for one second. Listeners were asked to keep their head still while the LED was lit. After the LED was turned off, the chair was rotated clockwise or anticlockwise at 5,10 , or $20^{\circ} / \mathrm{s}$. At a head position facing $\pm 15^{\circ}\left(+15^{\circ}\right.$ for clockwise rotation and $-15^{\circ}$ for anticlockwise rotation), a sound stimulus was presented from one loudspeaker randomly selected from the 13 loudspeakers within $\pm 15^{\circ}$ of the listener physical straight ahead (clockwise: 13 loudspeakers within $+15 \pm 15^{\circ}$, anticlockwise: 13 loudspeakers within $-15 \pm 15^{\circ}$, in world coordinates). Then, 
the chair was rotated to $\pm 30^{\circ}$ after the sound stimulus was presented.

We did not use any special devices to restrict the listeners' head movement, but their head position was observed by a magnetic sensor (Polhemus, Fastrak) attached to the top of the head at a sampling rate of $120 \mathrm{~Hz}$. The chair-rotation condition comprised 20 sessions and 1,560 trials in total (two rotational directions $\times$ three rotation speeds $\times 13$ loudspeaker positions $\times 20$ trials). Rotation directions, rotation speeds, and loudspeaker positions were presented in fully randomized order. Before test trials, listeners completed 36 practice trials.

This study was approved by the ethics committee of the Research Institute of Electrical Communication, Tohoku University. Written informed consent was obtained from each listener before participation in the study.

\section{RESULTS}

We measured fraction of left (0) or right (1) judgment for each condition. The point of subjective equality, which we call the point of subjective straight ahead (PSSA), and the just noticeable difference (JND) of PSSA for each listener were calculated numerically based on maximum likelihood fitting to psychometric functions $(0.5 \times$ binomial distribution function +0.5 ). PSSA was defined as the $50 \%$ point on the psychometric function, i.e., listeners were assumed to respond 50\% "left" and 50\% "right" across trials. JND was defined as the detection threshold yielding $75 \%$ correct responses in the $2 \mathrm{AFC}$.

Figure 2 depicts the average PSSAs and standard errors. One-way repeated-measure analysis of variance (ANOVA) was applied to PSSA with rotation speed as a factor. Four rotation speed levels were used (chair-still: $0^{\circ} / \mathrm{s}$, chair-rotation: 5,10 , and $20^{\circ} / \mathrm{s}$ ). The PSSA was more correctly estimated under the chair-still condition $(M=$ $-0.07)$ than under the chair-rotation condition $\left(5^{\circ} / \mathrm{s}\right.$, $\left.M=-0.55 ; 10^{\circ} / \mathrm{s}, M=-0.35 ; 20^{\circ} / \mathrm{s}, M=-0.37\right)$, but the rotation speed did not have any significant effect $(F(3,21)=0.66, n . s)$.

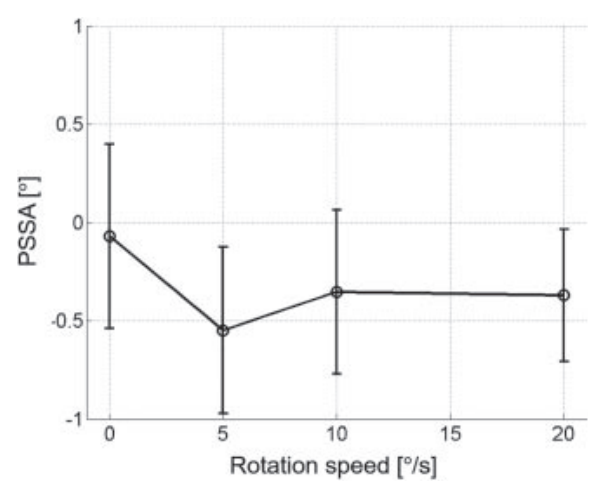

Fig. 2 Average PSSAs and standard errors.

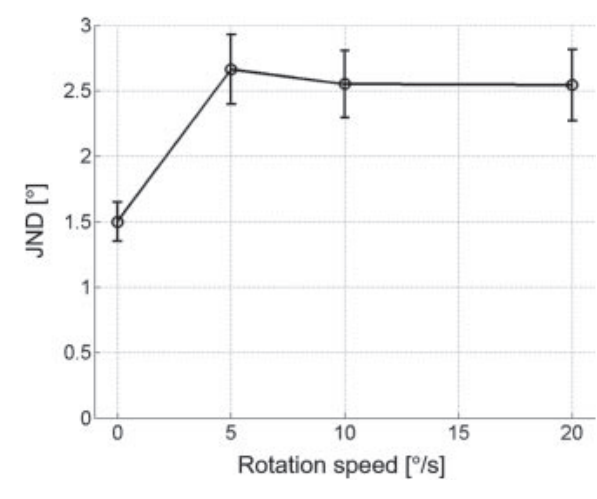

Fig. 3 Average JNDs and standard errors.

Figure 3 shows the average JNDs and standard errors. One-way repeated-measure ANOVA was applied to JND with the rotation speed as a factor. As in the case of PSSA, four rotation speed levels were used. The obtained results showed that the main effect of rotation speed was significant $(F(3,21)=7.77, p<0.01)$, and post-hoc analysis (Ryan's method, $p_{\mathrm{s}}<0.05$ ) revealed that JND was smaller for the chair-still condition $(M=1.50)$ than for the chair-rotation condition $\left(5^{\circ} / \mathrm{s}, M=2.66 ; 10^{\circ} / \mathrm{s}, M=2.55\right.$; $20^{\circ} / \mathrm{s}, M=2.54$ ). No significant difference was found between rotation speeds of 5,10 , and $20^{\circ} / \mathrm{s}$.

\section{DISCUSSION}

Earlier studies demonstrated that listener head/body movement facilitates sound localization [2-8], but recent reports have shown that sound localization accuracy deteriorates during listener head rotation [9-11]. Herein, we used a digitally controlled spinning chair to examine whether passive whole-body rotation affects sound localization accuracies of listener SSA and additionally investigated the effects of rotation velocity.

The obtained results showed that sound localization accuracies, in terms of PSSA detection thresholds for all rotation speeds, were significantly higher under the chairrotation condition than under the chair-still condition, while chair rotation speed had no significant effect. These findings indicated that both active [9-11] and passive head movement commonly results in the deterioration of sound localization accuracy, additionally suggesting that the velocity of whole-body rotation does not significantly impact sound localization accuracy. Therefore, these results can be regarded as consistent with those reported by Honda et al. [11], who also studied sound localization during active head movement.

Importantly, PSSA accuracy was not largely influenced by whole-body rotation velocity. This fact implies that the deterioration cannot be adequately explained by the blurring of ear inputs induced by listener head movement. At the highest rotation speed of $20^{\circ} / \mathrm{s}$, the listener rotates 
by $0.6^{\circ}$ during the sound stimulus presentation $(30 \mathrm{~ms})$. Since this disturbance can be regarded as continuous uniform distribution, its standard deviation becomes $\left(0.6^{2} / 12\right)^{0.5}=0.173^{\circ}$, which is much smaller than the JND values of $\sim 2.5^{\circ}$.

Thus, the deteriorating effects of head rotation on sound localization [9-11] might be not due to the bottomup effect such as the blurring of ear input but is rather a consequence of top-down effects such as the movement perception of listeners. For instance, Teramoto et al. [18] investigated whether visual linear self-motion information can alter auditory space perception, employing large-field visual motion to induce a self-motion perception with constant accelerations and a constant velocity in either a forward or backward direction. During the participants' experience of self-motion, a short noise burst was delivered from one of the loudspeakers aligned parallel to the motion direction along a wall to the left of the listener. The results showed that the sound position aligned with the subjective coronal plane was significantly displaced in the direction of self-motion, especially under the backward self-motion condition, as compared to the no-motion condition. Thus, self-motion information, irrespective of its origin, was demonstrated to be crucial for auditory space perception, which, in combination with the results presented herein, implies that several phenomena of auditory space perception might be well explained by the top-down information processing mechanism. These implications might contribute to a better understanding of spatial hearing mechanisms and the multisensory integration process.

At this point, several limitations of this study must be acknowledged. Although the room was kept dark during the experiment, listeners were able to use certain visual and positional cues while the LED was lit. Future studies must investigate whether these visual cues related to auditory information contribute to sound localization accuracy during passive whole-body rotation. Furthermore, in this study, we observed that sound localization accuracy was independent of listener passive head turn speed in the range of 5 to $20^{\circ} / \mathrm{s}$, and future work is expected to determine whether similar results can be obtained at even smaller speeds of $<5^{\circ} / \mathrm{s}$.

\section{ACKNOWLEDGMENTS}

This work was supported by JSPS Grant-in-Aid for Scientific Research (A) (No. 16H01736). A part of this work was conducted under the Cooperative Research Project Program of the Research Institute of Electrical Communication, Tohoku University (No. H29/A22).

\section{REFERENCES}

[1] W. A. Yost, X. Zhong and A. Najam, "Judging sound rotation when listeners and sounds rotate: Sound source localization is a multisystem process," J. Acoust. Soc. Am., 138, 3293-3310 (2015).

[2] H. Wallach, "On sound localization," J. Acoust. Soc. Am., 10, 270-274 (1939).

[3] A. Honda, H. Shibata, J. Gyoba, K. Saitou, Y. Iwaya and Y. Suzuki, "Transfer effects on sound localization performances from playing a virtual three-dimensional auditory game," Appl. Acoust., 68, 885-896 (2007).

[4] A. Honda, H. Shibata, S. Hidaka, J. Gyoba, Y. Iwaya and Y. Suzuki, "Effects of head movement and proprioceptive feedback in training of sound localization," i-Perception, 4, 253264 (2013).

[5] A. Honda, S. Tsunokake, Y. Suzuki and S. Sakamoto, "Effects of listener's whole-body rotation and sound duration on horizontal sound localization accuracy," Acoust. Sci. \& Tech., 39, 305-307 (2018).

[6] J. Kawaura, Y. Suzuki, F. Asano and T. Sone, "Sound localization in headphone reproduction by simulating transfer functions from the sound source to the external ear," J. Acoust. Soc. Jpn. (E), 12, 203-216 (1991).

[7] Y. Iwaya, Y. Suzuki and D. Kimura, "Effects of head movement on front-back error in sound localization," Acoust. Sci. \& Tech., 24, 322-324 (2003).

[8] S. Perrett and W. Noble, "The contribution of head motion cues to localization of low-pass noise," Percept. Psychophys., 59, 1018-1026 (1997).

[9] J. Cooper, S. Carlile and D. Alais, "Distortions of auditory space during rapid head turns," Exp. Brain Res., 191, 209-219 (2008).

[10] J. Leung, D. Alais and S. Carlile, "Compression of auditory space during rapid head turns," Proc. Natl. Acad. Sci. USA, 105, 6492-6497 (2008).

[11] A. Honda, K. Ohba, Y. Iwaya and Y. Suzuki, "Detection of sound image movement during horizontal head rotation," $i$ Perception, 7, 2041669516669614 (2016).

[12] A. W. Mills, "On the minimum audible angle," J. Acoust. Soc. Am., 30, 237-246 (1958).

[13] G. Lester and R. B. Morant, "Apparent sound displacement during vestibular stimulation," Am. J. Psychol., 83, 554-566 (1970).

[14] J. Lewald and H. O. Karnath, "Sound lateralization during passive whole-body rotation," Eur. J. Neurosci., 13, 22682272 (2001).

[15] W. Teramoto, S. Sakamoto, F. Furune, J. Gyoba and Y. Suzuki, "Compression of auditory space during forward selfmotion," PLoS One, 7, e39402 (2012).

[16] H. O. Karnath, D. Sievering and M. Fetter, "The interactive contribution of neck muscle proprioception and vestibular stimulation to subjective "straight ahead" orientation in man," Exp. Brain Res., 101, 140-146 (1994).

[17] G. Kerkhoff, F. Artinger and W. Ziegler, "Contrasting spatial hearing deficits in hemianopia and spatial neglect," Neuroreport, 10, 3555-3560 (1999).

[18] W. Teramoto, Z. Cui, S. Sakamoto and J. Gyoba, "Distortion of auditory space during visually induced self-motion in depth," Front. Psychol., 5, 848 (2014). 